\title{
System Level Simulation for Femtocellular Networks
}

\author{
S. Xing, P. Ghosal, K. Sandrasegaran and A. Daeinabi \\ Centre for Real-Time Information Networks, Faculty of Engineering and Information Technology, \\ University of Technology, Sydney, \\ Australia.
}

\begin{abstract}
LTE is an emerging wireless data communication technology to provide broadband ubiquitous Internet access. Femtocells are included in 3GPP since Release 8 to enhance the indoor network coverage and capacity. System level simulation is used for performance evaluation of LTE-Femtocellular networks. Research works on performance optimization could not be justified since there was no common reference simulator to do so until the inception of LTE-Sim. The simulation scenarios for Femtocells in LTE-Sim encompasses two-tier macro-femto scenario but to the best of our knowledge there is no published work on coding and scripting of femtocell scenario in LTE-Sim. In this paper, the development of a femtocell scenario is discussed with simulation outcomes.
\end{abstract}

Keywords-Adaptive Modulcation and Coding (AMC), Channel Quality Indicator (CQI), Digital Subscriber Line (DSL), Evolved Packet Core(EPC), evolved-Universal Terrestrial Radio Access Network (e-UTRAN), Exponential Proportional Fair (ExPF), Femtocell Access Point(FAP), Femtocell User Equipment (FUE), Macro eNodeB (MeNB), Macrocell User Equepment (MUE), Moblity Management Entity (MME), Modified Largest Weighted Delay First (MLWDF) Proportional Fair(PF), Physical Layer (PHY), Quality of Service(QoS), Radio Resource Management (RRM), Signal to Interference Plus Noise Ratio (SINR).

\section{INTRODUCTION}

In the next generation wireless communication systems, the primary challenge is to improve the indoor coverage, capacity enhancement as well as to provide users the mobile services with high data rates in a cost effective way. Performance of mobile system can be enhanced by evolving to emerging broadband technologies such as WiMAX [1] and LTE [2] but this may not be able to endure the exponential rise in traffic volume. These advancements in $4 \mathrm{G}$ physical layer (PHY) are approaching to the Shannon limit [3] and ensure maximum achievable data rate. So, further enhancement either in the PHY layer or available spectrum will not be adequate to overcome the coverage and capacity challenge. One of the approaches of solving this capacity and coverage related problem includes moving the transmitters and receivers closer to each other. This method loses its ground on economic feasibility because of deploying more base stations (BSs). Thus, small cells generally known as Femtocells with restricted access permission to fewer users compared to macrocell are chosen by the mobile operators as a possible solution to improve the network coverage, especially to the indoor users with ubiquitous high speed connectivity. These Femtocell base stations are referred to as Femto Access Points (FAPs). They have a short-range $(10-30 \mathrm{~m})$ and require a low power $(10$ $100 \mathrm{~mW}$ ) [5] to provide high-bandwidth wireless communication services in a cost effective way. Femtocells incorporated with the plug and play capabilities work in mobile operator owned licensed spectrum and enable Fixed Mobile Convergence (FMC) [ $\underline{6}$ ] by connecting to the core network via broadband communications links (e.g., DSL). Unlike macrocells, FAPs are typically installed and maintained by the end users in an unplanned manner. This uncoordinated nature of femtocell deployment poses challenges on radio resource management (RRM). Existing LTE/LTE-A macrocell system approach to face interference coordination, frequency planning, access policies and radio resource scheduling do not necessarily apply in a femtocell scenario [3]. Thus, there is a need to develop RRM techniques that are more suited for femtocell scenarios. In this perspective, it is important to find an accurate simulation framework. The current open-source simulation tools are only able to simulate parts of the LTE system [3]. At present, we are not aware of any simulator that simulate femtocell along with the media access control (MAC) and PHY layer models with handover strategies and propagation loss models for indoor environments. To bridge this gap, this paper describes our efforts to build a single femtocell and multi user environment in the existing open source LTE-Sim framework [7] encompassing scenarios with both macro and femto cells. The rest of the article is organized as follows: Section II describes the LTE Network Architecture and Femtocells. Section III describes the modeling and coding of Femtocells in LTE-Sim. Section IV shows the simulation result of the FAP model. Finally, Section V draws the conclusion.

\section{LTE FEMTOCELL: NETWORK ARCHITECTURE}

\section{A. LTE and OFDMA Frame Structure}

The 3GPP, LTE is packet-switched with flat architecture and mainly composed of three parts: the UE, the e-UTRAN, and the packet switched core network (EPC). EPC is responsible for all services provided to UE including voice and data to the user using packet switching technology. e-UTRAN has only one node i.e., the evolved NodeB (eNB) which handles the radio communication between UE and EPC. The physical layer of radio interface supports both time (TDD) and frequency (FDD) division duplexing. High flexibility in scheduling and interference management is achieved by narrow, mutually orthogonal subcarriers through the use of 
Orthogonal Frequency Division Multiple Access (OFDMA) [2]. In time domain, radio resources are allocated in $1 \mathrm{~ms}$ (Transmission Time Interval -TTI).10 consecutive TTIs form the LTE frame lasting $10 \mathrm{~ms}$. The $10 \mathrm{~ms}$ radio frame consists 10 equally spaced sub-frames where each sub-frame is divided in two $0.5 \mathrm{~ms}$ successive slots and each slot consists seven OFDM symbols with normal cyclic prefix. In the frequency domain, whole bandwidth is divided into $180 \mathrm{KHz}$ sub-channels where each composed of 12 consecutive sub-carriers. A sub-carrier of $15 \mathrm{KHz}$ that spans over $0.5 \mathrm{~ms}$ in time domain is called the Resource Element (RE). Resource Block (RB) is the smallest radio resource that is used for data transmission by UE in time/frequency domain, spans one $0.5 \mathrm{~ms}$ time slot in the time domain and over one sub-channel in the frequency domain. The number of sub-channels varies accordingly to different system bandwidth configurations due to the fixed sub-channel size (e.g., 25 and 50 RBs for system bandwidths of 5 and $10 \mathrm{MHz}$, respectively).

\section{B. Femtocell and Its challenges}

Femtocells not only boost indoor coverage [9] and capacity but also improve battery life of UEs since UE doesn't need to communicate with a distant macrocell base stations. Fig. 1 shows the basic two tier macro-femto network architecture. FAPs which have a less computational capability [3], are connected through DSL (Digital Subscriber Lin) in indoor scenarios. The LTE macro system based on flat architecture connects all the eNBs through X2 interface and

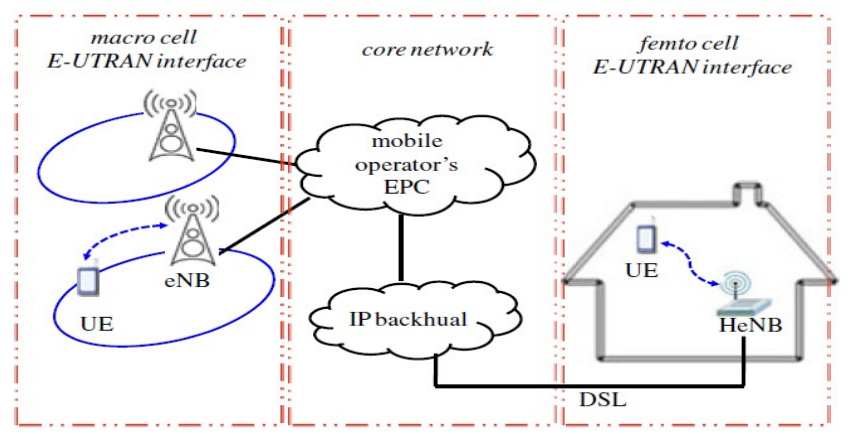

Figure 1: Two-tier macro-femto network architecture [3]

the RRM procedure is done by eNB. Unlike the LTE macrocell system the FAPs lack the X2 interface among the femtocells. Thus, the benefits of femtocells are jeopardized by this uncoordinated nature and come with the cost of increased complexity in spectrum management. Interference Coordination [10], Radio Resource Management (RRM) and HO management [17] is expected to become more complex with the unplanned and vast deployment of LTE femtocells without the centralized frequency planning. FAPs encounter two types of interferences: co-layer and cross-layer [10]. Colayer interference occurs with the dense deployment of femtocells when users served by one FAP are interfered by neighboring FAPs. On the other hand, cross-layer interference is generated when femtocells and macrocells share the same portion of the spectrum within a specific area. Several innovative solutions for mitigating the cross-layer intercell interference are proposed in [11-14]. Femtocells can to operate in one of three access modes, i.e., closed access mode, open access mode or hybrid access mode [15]. Closed access mode is generally deployed in residential scenarios and a group of registered users called Closed Subscriber Group (CSG) have the permission to access the femtocell. Users without the access permission suffer the impact of the interference generated by FAPs [16] in closed access mode. In case of open access mode, any UE can access the femtocell and benefit from its services but when it comes to resource usage, congestion and security, open access is not a suitable choice. In hybrid access mode, a limited number of unknown MUE may access the femtocell while a fixed number of users defined by the owner can access the femtocell ubiquitously but may suffer the risk of security breach [16].

\section{LTE-SIM: FEMTOCELL MODELLING}

All the aforementioned open issues lead to a unified modelling approach to LTE femtocells in LTE-Sim. The key aspects of LTE-Sim proposed in [7] are briefly summarized below:

\section{Basic Background}

LTE-Sim is an event-driven simulator written in C++ using the well-known object-oriented platform [7]. The four main modules of LTE-Sim are: a) the Simulator, b) the NetworkManager, c) the Flowsmanager and d) the FrameManager. Each network node of LTE architecture is implemented through different class in this simulator (i.e eNB class, UE class, MME class etc). Several functionalities of LTE networks, including the models of e-UTRAN and EPC, DL and UL transmissions, QoS management, user mobility, handover procedures, and frequency reuse techniques are provided by the ProtocolStac class. The entire LTE protocol stack is composed of three network nodes, i.e., UE, eNB, and MME to form application to PHY layer including radio link control(RLC), radio resource control(RRC) and MAC entities. These network nodes can either be destination or source maintain their flow of data through source and destination IP addresses, ports and transport protocol. In addition LTE-Sim platform supports well known scheduling strategies (eg., PF, MLWDF, ExPF and Log rules) and AMC schemes [7]. This simulator also supports CQI feedback by converting the channel quality estimation report from UE and converting it to set of CQI feedbacks reported to eNB. and several other features. In the application layer of LTE-Sim, four traffic generators have been developed(trace-based, on-off, infinite buffer and constant bit rate).Packet transmission and propagation loss models are covered by the simulator Channel module [7] using four different phenomena as suggested in [19]: the path loss, the penetration loss, the shadowing and the fast fading due to the signal multipath. In LTE-Sim, the path loss calculation for macro users inside buildings considers additional attenuation factor due to the presence of external walls (default value of the external wall attenuation is $20 \mathrm{~dB}$ [18] and WinnerII channel model [20] is also considered for residential FAP users. All the aforementioned features give it the flexibility and modularity to device a complete system for simulating LTE femtocells. To build a Femtocell scenario in existing LTE-Sim platform the steps are: $i$. Create Femtocell Scenario and ii. Create Femtocell User. 


\section{i) Create Femtocell Scenario:}

Creating Femtocell Scenario is constructing FAP inside buildings. FAPs and eNBs are identified by unique IDs and its position is defined by Cartesian coordinate system [7]. UE information such as CQI feedbacks, uplink channel quality, and uplink scheduling request are managed by ID and position tracking. In order to form Femtocell scenario, the information of number of buildings, locations of the building with FAP, building type, location of FUE etc. have to be defined in Femtocell Scenario header file(SingleCellWithFemto.h) of LTE-Sim. Code Block 1. shows how to create a building with position(x:-400,y: 400). Likewise, the number of the buildings can be increased according to the environment (i.e. urban, dense urban or residential etc.)

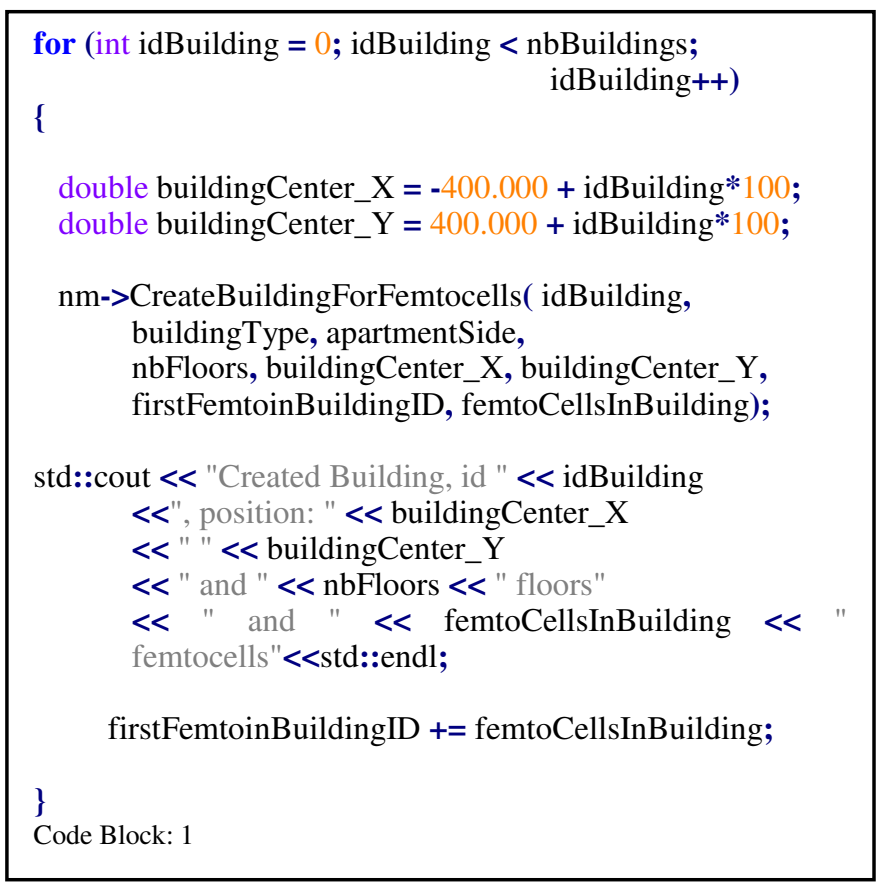

Building is composed of a number of apartments and in this simulation each one defines the area of a given femtocell. According to the definition in [18], two different types of building is developed and used in LTE-Sim: (a) Dual Stripe blocks and (b) $5 \times 5$ apartment grid. Dual Strip blocks consists two building rows each with 10 . The latter is a building of 25 apartments located over a $5 \times 5$ grid. This apartment grid can also be modified according to the traffic environment. In this paper, $3 \times 3$ apartment grid as Fig. 2 is considered. Each apartment has a length and width of 10 meters to form a squared area of $100 \mathrm{~m}^{2}$. In this paper, each apartment with one active femtocell is considered. This means that, for instance, a building with $3 \times 3$ apartment grid can contain up to 9 femtocells. Grid of apartments inside of the building could be defined by configuring the grid settings (i.e. building types) in the header file (SingleCellWithFemto.h). Building walls are formed in NetworkManager.cpp where, function NetworkManager::CreateBuildingForFemtocells() gets building ID defined in SingleCellWithFemto.h and builds up the building according to the building type as presented in Fig. 2 .

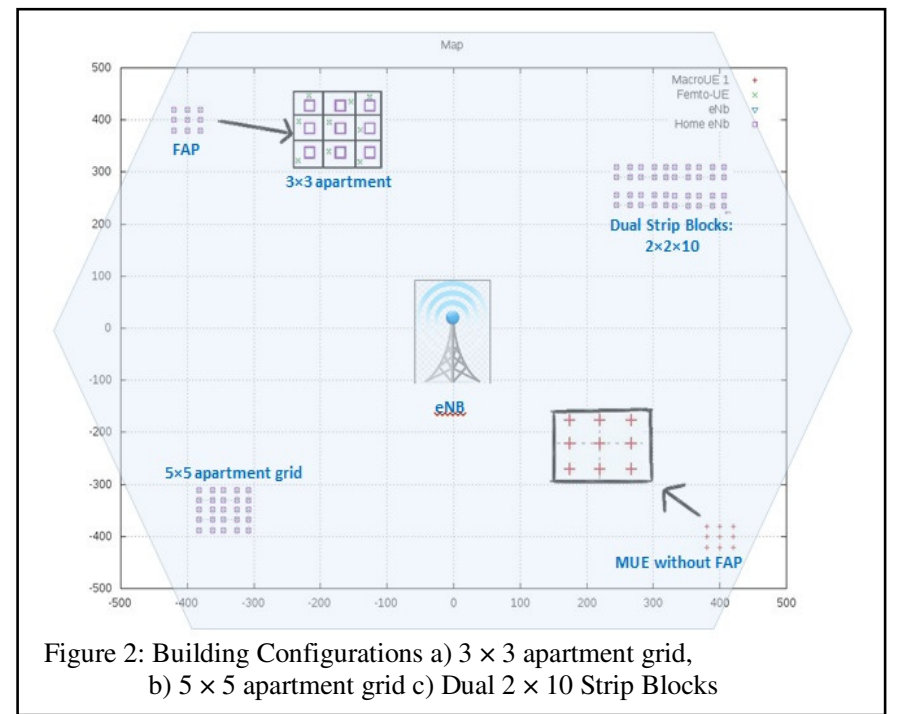

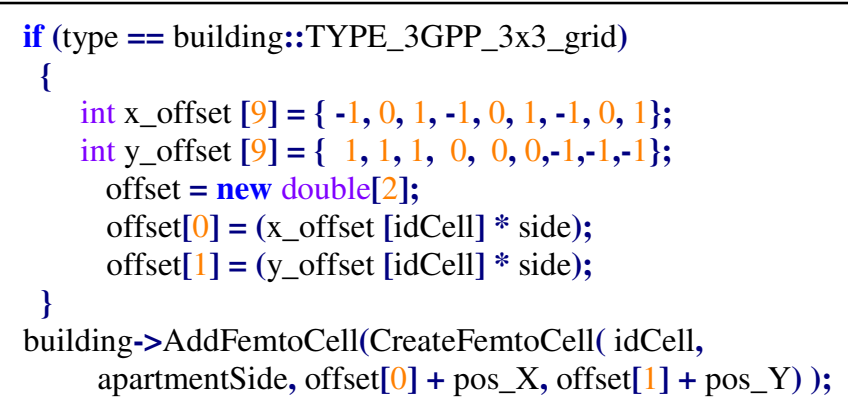

Code Block 2

Location of each femtocell inside the building is defined in IndoorScenario.h and the center of each building denotes the origin of each FAP. Array of x_offset and y_offset denotes the coordinates of each FAP, inside $3 \times 3$ apartment grid (i.e. building type 1) in Code Block 2 .

vector $<$ CartesianCoordinates $*>$ *positions $=$

GetUniformUsersDistributionInFemtoCell (idCell, nbFemtoUE);

for (int $\mathrm{i}=0$; $\mathrm{i}<$ nbFemtoUE; $\mathrm{i}++$ )

\{

//FUE's random position

double posX = HeNBs->at (j)->GetMobilityModel ()$>$ GetAbsolutePosition ()->GetCoordinateX (); double $\operatorname{pos} \mathrm{Y}=$ HeNBs- $>$ at (j) $>>$ GetMobilityModel $($ )$>$ GetAbsolutePosition ()->GetCoordinateY (); double speedDirection $=($ double $)($ rand ()$\% 360)$

$*(2 * 3.14) / 360)$;

UserEquipment* ue $=$ new UserEquipment (idUE,

posX, posY, speed, speedDirection, femtocells->at (j), HeNBs->at (j), $0, / / \mathrm{HO}$ deactivated!

Code Block 3 


\section{ii) Create Femtocell User(FUE):}

The number of FUEs is set up at the beginning of the simulation in shell script. Code blocks 3 demonstrates to set up a loop to generate one coordinate for each FUE and then call the "new UserEquipment()" function to pass the coordinate variables to the FUE with ID "i". By taking over the position coordinate "pos_X, pos_Y" to each FUE creation function within the loop, all the FUE will obtain an individual coordinate. In this case, FUEs are obtaining the coordinate that as same as the FAP. Fig. 3 shows the modified class diagram of Femtocell Scenario in LTE-Sim.

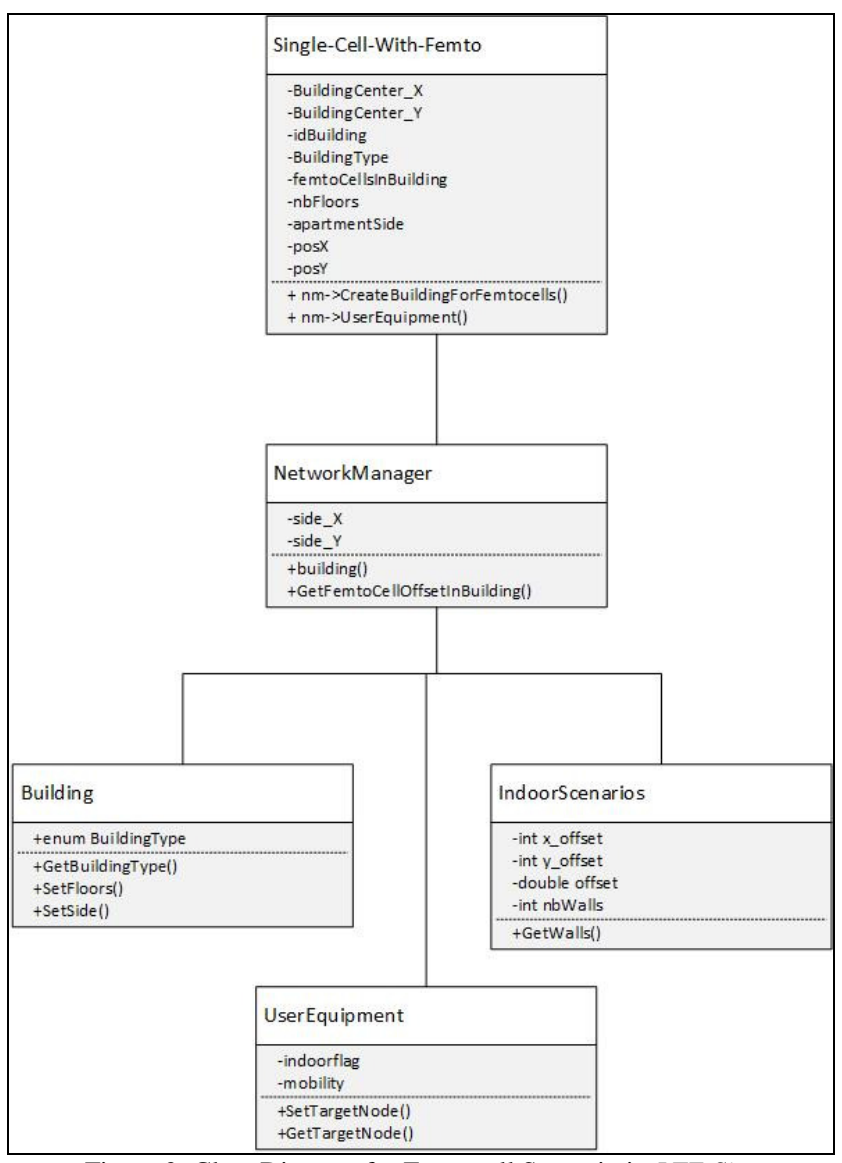

Figure 3: Class Diagram for Femtocell Scenario in LTE-Sim

\section{Simulation Results}

In this section, some simulation outcomes regarding the change in throughput of UE and scalability in the presence of FAP are highlighted. The simulation results show the performance gains with the deployment of femtocells in a $3 \times 3$ urban scenario using different scheduling algorithms (PF, MLWDF, EXP/PF). Main simulation parameters are summarized in Tab. 1. In this paper it is investigated that the impact of the femtocell deployment in a scenario where an eNB operates in $20 \mathrm{MHz}$ bandwidth is located at position $(0$, 0 ) and transmits using an omni-directional antenna. Two different cases are object of the study:

a) Traditional urban environment where coverage is provided by one macrocell and without the presence of femtocells is taken as reference case. b) Urban environment where femtocells are sharing the same portion of available spectrum with macrocells are considered to be active in each apartment.

Simulations were carried out with fixed number of static users, each user is experiencing real-time downlink single flow (either VoIP or Video) from an infinite buffer source. Fig. 4-6 show the average user throughput (VoIP and Video) with and without the presence of Femtocells using different .

Table 1: Simulation Parameters

\begin{tabular}{|l|l|}
\hline Bandwidth & $20 \mathrm{MHz}$ \\
\hline Frame Structure & FDD \\
\hline eNb power transmission & $43 \mathrm{dBm}$ \\
\hline FAP power transmission & $20 \mathrm{dBm}$ \\
\hline CQI & $\begin{array}{l}\text { Full Bandwidth and periodic } \\
(2 \mathrm{~ms}) \text { reporting scheme }\end{array}$ \\
\hline Apartment Size & $100 \mathrm{~m}^{2}$ \\
\hline Buildings & 1 \\
\hline Users & 3 per HeNB \\
\hline Scheduler & PF, M-LWDF, EXP-PF \\
\hline Traffic & VOIP, VIDEO \\
\hline
\end{tabular}

Packet Schedulers-PF (Proportional Fair), M-LWDF (Maximum-Largest Weighted Delay First) and EXP/PF(Exponential-Proportional Fair).

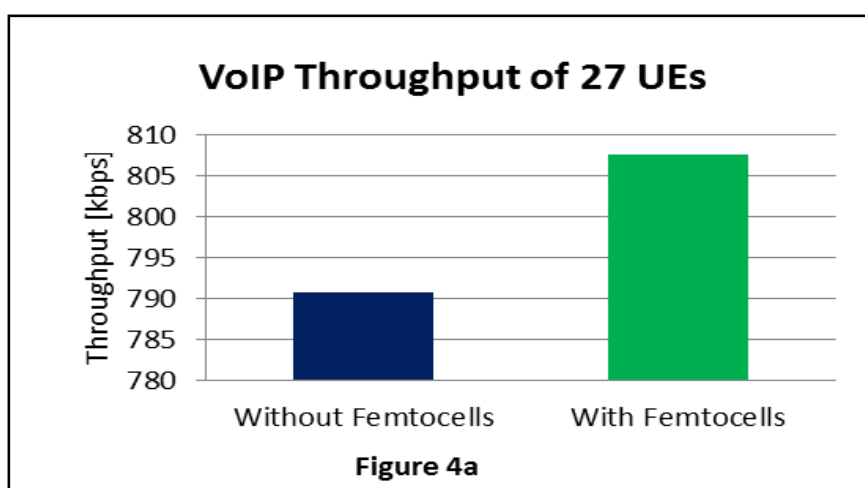

\section{VIDEO Throughput of 27 UEs}

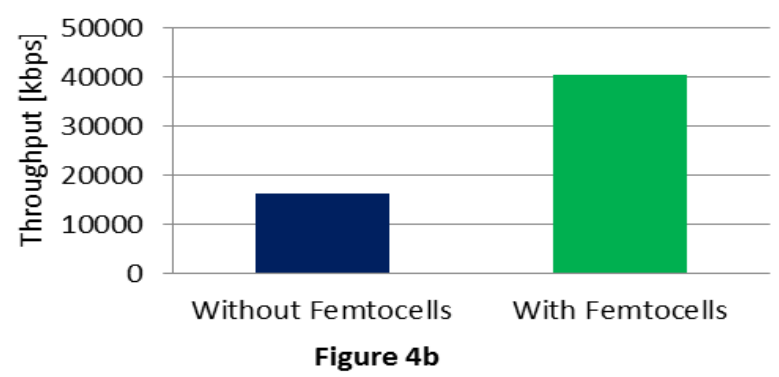

Figure 4: a) VoIP Throughput comparision b) Video throughput comparison of 27 UEs with PF Scheduling 

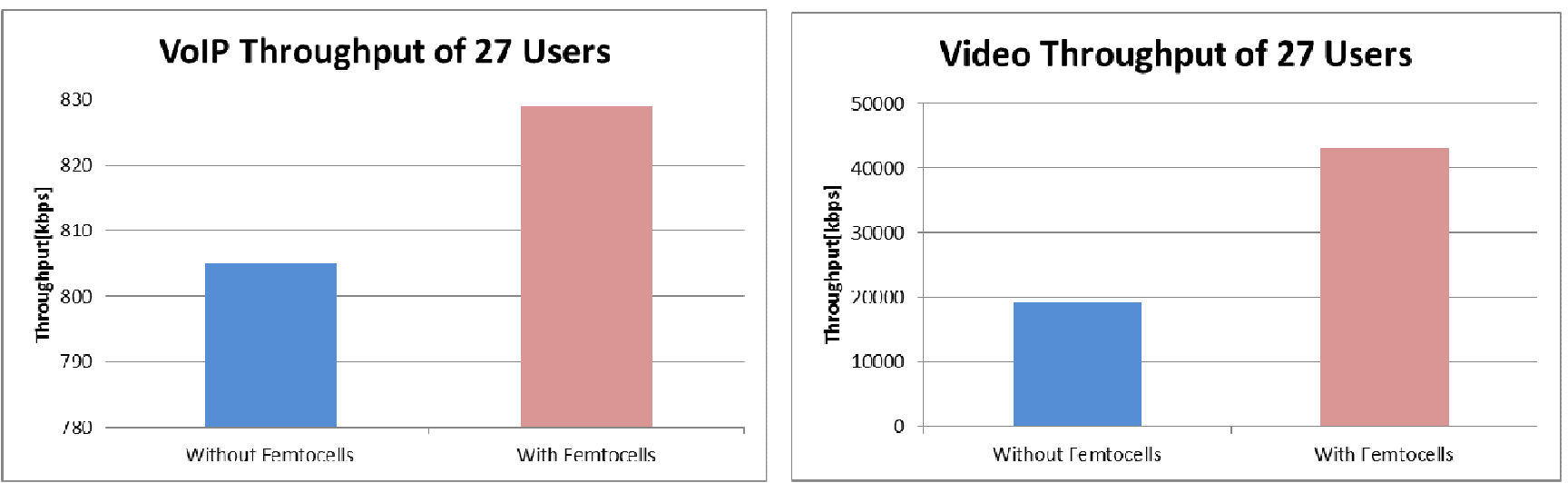

Figure 5: a) VoIP Throughput comparision b) Video throughput comparison of 27 UEs with M-LWDF Scheduling
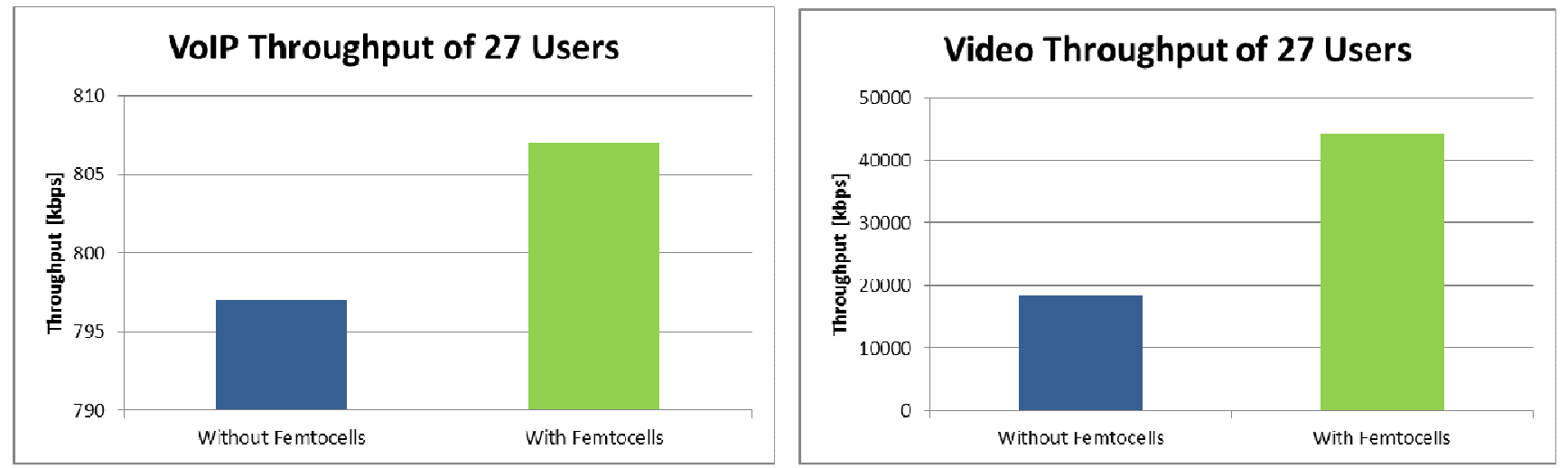

Figure 6: a) VoIP Throughput comparision b) Video throughput comparison of 27 UEs with EXP-PF Scheduling

In the simulation the building $(-400,400)$ was located 566 meters north west from the eNB. In the scenario without femtocells, cell edge macrocell indoor users suffer poor coverage as well as low channel quality due to the strong influence of path loss and penetration loss. Significant raise in network average throughput (VoIP and Video) was observed with the inception of femtocells inside the building. From Fig. 4 it is seen that, among all the schedulers, PF has the lowest VoIP and Video throughput since this channel aware scheduler doesn't consider QoS requirements while scheduling decisions, making it not suitable for real-time traffic applications. Channel aware/QoS aware scheduler MLWDF has the highest average VoIP throughput (Fig.-5) whereas Fig. 6 shows EXP/PF scheduler which considers PF rule and exponential function of the end-to-end delay has highest average Video throughput.

\section{CONCLUTION}

In this article, demonstration to simulate femtocell scenarios with features and models compliant with 3GPP specifications in LTE-Sim framework is provided. The femtocell scenario shown in this paper can be used for investigating performances in a wider range that can differ in terms of network layouts (i.e., number and position of base- stations, building, and streets), system load (i.e., number of users and applications) and so on. As a future study, we plan to enhance the LTE-Sim Femtocell framework with the aim of implementing mobility management, handover, radio resource allocation schemes [21] as well as other algorithms and protocols based on both self-organizing network (SON) and cognitive paradigm believing the extreme modularity of $L T E$ Sim.

\section{REFERENCES}

[1] L. Nuaymi, WiMAX: Technology for Broadband Wireless Access. Wiley, NewYork, 2008.

[2] E Dahlman, S Parkvall, J Skold, P Beming, 3G Evolution HSPA and LTE for Mobile Broadband. Academia Press, USA, 2008.

[3] F. Capozzi, G. Piro, L. Grieco, G. Boggia, P. Camarda, "On Accurate simulations of LTE femtocells using an open source simulator," in EURASIP Journal on Wireless Communication and Networking, 2012.

[4] Onyeije Consulting LLC, Solving the capacity crunch (2011).

[5] T. Zahir, K. Arshad, A. Nakata, K. Moessner,"Interference Management in Femtocells," in Commun. Surveys \& Tutorials, vol. 15, pp. 293-311, 2013.

[6] www.en.wikipedia.org/wiki/Fixed_mobile_convergence/.

[7] G Piro, L Grieco, G Boggia, F Capozzi, P Camarda,’Simulating LTE cellular systems: an open-source framework, " in Vehicular Technology, IEEE Trans. vol. 60, pp. 498-513, 2011. 
[8] 3GPP TS 36.211,'Radio Access Network - Physical Channel and Modulation (Release 8)," 2008.

[9] L. 3GPP TS 22.220," Radio Access Network - Service requirements for Home Node B (HNB) and Home eNode B (HeNB)," 2011.

[10] D. Lopez-Perez, I. Guvenc, G. de la Roche, M. Kountouris, T. Q. S. Quek, and Z. Jie, "Enhanced intercell interference coordination challenges in heterogeneous networks," IEEE Wireless Commun., vol. 18, pp. 22-30, 2011.

[11] N. Saquib, E. Hossain, L. Long Bao, and K. Dong, "Interference management in OFDMA femtocell networks: issues and approaches," IEEE Trans. Wireless Commun., vol. 19, pp. 86-95, 2012.

[12] A. Damnjanovic, J. Montojo, W. Yongbin, J. Tingfang, L. Tao, M. Vajapeyam, et al., "A survey on 3GPP heterogeneous networks," IEEE Wireless Commun. , vol. 18, pp. 10-21, 2011.

[13] S. Iwelski, B. Zijian, G. H. Bruck, P. Jung, B. Badic, T. Scholand, et al., "Analysis of interference-aware receivers in heterogeneous LTE networks," in Int. Conf. Commun. in China (ICCC), IEEE/CIC, China, pp. 314-320, 2013.

[14] W.Yi, Z. Dongmei, J. Hai and W. Ye, " A Novel Spectrum Arrangement Scheme for Femtocell Deployment in LTE Macrocells," Proc. IEEE $20^{\text {th }}$ Symp. on Personal, Indoor and Mobile Radion Commun., pp. 6-11, 13-16, Sept. 2009.

[15] R. Bendlin, V. Chandrasekhar, C. Runhua, A. Ekpenyong, and E. Onggosanusi, "From homogeneous to heterogeneous networks: A 3GPP Long Term Evolution rel. 8/9 case study," in 45th Annu. Conf., Inform. Sci. and Syst. (CISS), Baltimore, MD, pp. 1-5, 2011.

[16] H. Mahmoud, I .Guvenc,” A comparative study of different deployment modes for femtocell networks," in IEEE Int. Symposium on Personal, Indoor and Mobile Radio Commun., Palo Alto, USA, pp. 1-5, 2009.

[17] D. Xenakis, N. Passas, L. Merakos, C. Verikoukis, "Mobility Management for Femtocells in LTE-Advanced: Key Aspects and Survey of Handover Decision Algorithms" in IEEE Commun. Surveys \& Tutorials., vol. 16 pp. 2014.

[18] 3GPP, R4-092042, "Simulation assumptions and parameters for FDD HeNB RF requirements," 3GPP TSG RAN WG4 Meeting 51, 2009.

[19] 3GPP TR 25.814," Physical layer aspect for evolved Universal Terrestrial Radio Access (UTRA) (Release 7)," 2006.

[20] Winner, "WINNER II Channel Models - Deliverable,"www.istwinner.org/WINNER2-Deliverables/D1.1.2v1.2.pdf, $\quad$ D1.1.2 V1.2, 2007.

[21] F. Capozzi, G. Piro, L. Grieco, G. Boggia, P. Camarda, " Asystemlevel simulation framework for LTE Femtocell," in Proc. of International Conference on Simulation Tools and Techniques(ICST), Desenzano, Italy, pp.1-3, 2012. 